\title{
Asymmetry sum rule for molecular predissociation
}

\author{
F. T. Hawes and L. W. Torop \\ Department of Physics and Mathematical Physics, The University of Adelaide, Adelaide, South Australia 5005, Australia \\ B. R. Lewis and S. T. Gibson \\ Research School of Physical Sciences and Engineering, The Australian National University, \\ Canberra, Australian Capital Territory 0200, Australia \\ (Received 11 July 2000; published 13 December 2000)
}

\begin{abstract}
In the case of weak diatomic molecular predissociation by noninteracting, optically inactive continuum states, it is demonstrated that the predissociation line shape is more accurately represented by a Beutler-Fano profile than by a Lorentzian. The weak asymmetry that is found to occur is due principally to interactions with neighboring vibrational resonances. For this type of predissociation in the case of multiple continua, a sum rule for the corresponding line-shape asymmetry is derived. This sum rule is verified numerically using singlechannel and multichannel coupled Schrödinger-equation calculations for the Schumann-Runge band system of $\mathrm{O}_{2}$. Similar results are presented for the case of optically active continua.
\end{abstract}

DOI: 10.1103/PhysRevA.63.012513

PACS number(s): 33.70.Jg, 33.80.Gj

\section{INTRODUCTION}

Diatomic molecular predissociation, a process in which interaction between a bound state and a continuum leads to spectral broadening and shifting, together with the consequent production of atomic fragments, is an important process in the photochemistry of the terrestrial atmosphere. Weak predissociation is commonly treated theoretically using the Fano configuration-interaction (CI) framework [1-3] in which the predissociating resonance is characterized by (energy-dependent) width, shift, and line-shape parameters. In the case of a single bound state embedded in the continuum, and where the energy dependences of the parameters may be neglected, the resonance has a (symmetric) Lorentzian line shape in the case of an optically inactive continuum, or an (asymmetric) Beutler-Fano [1,4] line shape in the case of an optically active continuum. If the energy dependences of the parameters over the resonance cannot be neglected, then these formal (constant-parameter) line shapes do not apply [5,6], the Lorentzian, in particular, becoming asymmetric [5]. Furthermore, in cases where several bound states are embedded in the continuum, interactions between the resonances may provide an additional contribution to the line-shape asymmetry $[1,3,7]$. Treatments of such interactions between close-lying resonances have been reported for transitions to the different fine-structure components of an electronic state [8] and to vibrational levels of different electronic states [9].

Here, however, we are concerned principally with the weak predissociation of well-separated vibrational levels of an electronic state, where it may be assumed that the resonance widths are much smaller than the separation between adjacent resonances. This condition is usually associated with the isolated-resonance approximation (IRA), which reduces to the single-resonance case. However, since here we wish to consider the small effects of interactions between resonances, we call this condition the nearly-isolatedresonance approximation (NIRA).

Many real examples of predissociated band systems, e.g., those where the predissociation is spin-orbit induced, are in the weak-interaction, NIRA regime and involve optically inactive continua. Therefore, most simulations of such systems, with applications, e.g., in atmospheric photochemical models [10], have been based on the Lorentzian profile [11]. However, neglect of both the energy dependences of the lineshape parameters and the effects of interactions between resonances makes this line-shape assumption questionable away from the central part of the resonance. In fact, through the use of a coupled-channel Schrödinger equation (CSE) scattering-theory treatment, which is applicable for all energies in a vibrational sequence of predissociated levels, it has been suggested [12] that each resonance is described accurately to much greater distances from the resonance center by a Fano, rather than a Lorentzian, profile. This distinction is of particular importance, e.g., in the development of models of the absorption of solar vacuum-ultraviolet radiation in the terrestrial atmosphere, where it is the wings of the predissociated lines of the Schumann-Runge (SR) bands of $\mathrm{O}_{2}$ that control the penetration of radiation to the lowest altitudes [12].

Furthermore, many examples of predissociation involve interactions with multiple, mutually noninteracting continua. In the weak-interaction, IRA regime, CI [1,3] and analytic scattering-model [13] methods indicate that, when a bound state is coupled to a set of such continua, the width and shift contributions from each continuum are additive. These two "sum rules" for the width and shift have also been verified numerically by comparisons between the results of singlechannel and multichannel scattering-theory calculations for a number of real predissociation problems $[14,15]$. The validity of the sum rules makes it unnecessary to perform multichannel calculations, allowing, e.g., each individual interaction to be treated using the perturbative CI theory.

In this work, using the Fano-Mies formalism $[1,3]$ we confirm that the line shape for weak predissociation by noninteracting, optically inactive continua is more accurately represented by a Fano profile than by a Lorentzian. In addition, we suggest a sum rule for the line-shape asymmetry for 
this type of molecular predissociation in the case of multiple continua. This asymmetry sum rule is verified numerically using single-channel and multichannel CSE calculations for the SR band system of $\mathrm{O}_{2}$. Finally, similar results are presented for the case of optically active continua.

\section{LINE-SHAPE THEORY}

\section{A. The isolated resonance}

The Fano CI treatment [1,3] of an isolated diatomic predissociating resonance can be expressed in terms of the normalized discrete vibrational wave function $\phi$ in the bound diabatic potential-energy curve, the energy-normalized continuum radial wave functions $\mathcal{R}_{\alpha}$ in the dissociative diabatic potentials $(\alpha=1,2,3, \ldots)$ that cross the bound potential, the electronic coupling functions $h_{\alpha}$ between the bound electronic state and the dissociative electronic states $\alpha$, the normalized discrete vibrational wave function $\phi_{i}$ of the initial state in the photoabsorption process, and the electronic transition amplitudes $\tau$ and $\tau_{\alpha}$ from the initial to the bound and dissociative electronic states, respectively. In the Fano theory $[1,3]$, the continuum states are chosen as noninteracting, i.e., $\left\langle\mathcal{R}_{\alpha}\left(E^{\prime \prime}\right)\left|h_{\alpha \beta}\right| \mathcal{R}_{\beta}\left(E^{\prime}\right)\right\rangle=0$, where $h_{\alpha \beta}$ is the electronic coupling between continua $\alpha$ and $\beta$.

In the general case, where there is nonzero photoabsorption to at least one of the dissociative continua, the photodissociation cross section has the form $[1,3]$

$$
\sigma=\sigma_{d} \frac{(q+\epsilon)^{2}}{1+\epsilon^{2}}+\left(\sigma_{c}-\sigma_{d}\right),
$$

where the symbols are defined below. The dimensionless energy parameter

$$
\epsilon \equiv \frac{E-\left[E_{0}+\Delta(E)\right]}{\frac{1}{2} \Gamma(E)},
$$

where $E$ is the energy of the upper state in the photabsorption process, $E_{0}$ is the (unperturbed) energy of the bound state, and the "width function"

$$
\Gamma(E) \equiv 2 \pi V^{2}(E),
$$

where $V$ is the dissociation amplitude. In the case of noninteracting continua, $V$ satisfies the relation

$$
V^{2}(E)=\sum_{\alpha} V_{\alpha}^{2}(E)
$$

where $V_{\alpha}$ is the dissociation amplitude to continuum $\alpha$ :

$$
V_{\alpha}(E)=\left\langle\mathcal{R}_{\alpha}(E)\left|h_{\alpha}\right| \phi\right\rangle \text {. }
$$

The "shift function",

$$
\Delta(E) \equiv \frac{1}{2 \pi} \mathrm{P} \int d E^{\prime} \frac{\Gamma\left(E^{\prime}\right)}{E-E^{\prime}},
$$

where $\mathrm{P}$ indicates that the principal value should be taken. The profile function $[1,3]$

$$
q(E) \equiv \frac{\left\langle\phi|\tau| \phi_{i}\right\rangle+\sum_{\alpha} \mathrm{P} \int d E^{\prime} V_{\alpha}\left(E^{\prime}\right)\left\langle\mathcal{R}_{\alpha}\left(E^{\prime}\right)\left|\tau_{\alpha}\right| \phi_{i}\right\rangle /\left(E-E^{\prime}\right)}{\pi \sum_{\alpha} V_{\alpha}(E)\left\langle\mathcal{R}_{\alpha}(E)\left|\tau_{\alpha}\right| \phi_{i}\right\rangle} .
$$

$\sigma_{c}$ is the total cross section to all the continua; $\sigma_{d}$ is the cross section to that part of the energy- $E$ continuum space into which the bound state decays [16]:

$$
\frac{\sigma_{d}(E)}{\sigma_{c}(E)}=\frac{\left|\sum_{\alpha}\left[V_{\alpha}(E) / V(E)\right]\left\langle\mathcal{R}_{\alpha}(E)\left|\tau_{\alpha}\right| \phi_{i}\right\rangle\right|^{2}}{\sum_{\alpha}\left|\left\langle\mathcal{R}_{\alpha}(E)\left|\tau_{\alpha}\right| \phi_{i}\right\rangle\right|^{2}} .
$$

If the resonance is so narrow that $q, \Gamma, \Delta, \sigma_{d}$, and $\sigma_{c}$ can be taken to be constant over its peak, then the energy dependence of the cross section $\sigma$ defined by Eq. (1) is contained entirely in $\epsilon$, a linear function of energy. In this case, the resonance peak is said to have the Fano profile form, with profile index $q[1,3]$.

\section{B. Energy dependence}

If the continua are optically inactive (photoabsorption to each continuum is zero, i.e., $\left\langle\mathcal{R}_{\alpha}\left|\tau_{\alpha}\right| \phi_{i}\right\rangle=0$ for all $\alpha$ ), then $\sigma_{c}=0=\sigma_{d}$ and, if there is any absorption at all, $|q| \rightarrow \infty$ [see Eq. (7)]. Under these conditions, the CI procedure $[1,3]$ which leads to Eq. (1) in the case of optically active continua yields the following result for the photodissociation cross section:

$$
\sigma=\frac{\sigma_{b}}{\pi} \frac{\frac{1}{2} \Gamma(E)}{\left[E-\left(E_{0}+\Delta(E)\right]^{2}+\left[\frac{1}{2} \Gamma(E)\right]^{2}\right.},
$$

where the energy-independent factor $\sigma_{b}$ is the integrated photoabsorption cross section to the dissociating state in the 
bound diabatic potential. If $\Gamma$ and $\Delta$ can be taken to be constant over the peak, then the peak has a Breit-Wigner or Lorentzian line shape, with a maximum at energy $E=E_{0}$ $+\Delta$ and a full width at half maximum (FWHM) of $\Gamma$.

It has been demonstrated numerically by Bandrauk and Laplante [5] that the energy dependences of $\Gamma(E)$ \{and, consequently, of $\Delta(E)$ [see Eq. (6)]\} over a peak introduce an asymmetry into the Lorentzian line shape. Here, we characterize the approximate shape of the asymmetric line. In the particular case of interest to this work, weak energy variations over a narrow peak result in a slightly asymmetric, near-Lorentzian line shape. The energy dependence of $\Gamma(E)$ is, to a good approximation, determined by the energy dependences of the Franck-Condon factors $\left|\left\langle\mathcal{R}_{\alpha}(E) \mid \phi\right\rangle\right|^{2}$. Although these Franck-Condon factors may be oscillatory functions of energy [5], over a narrow peak it is reasonable to use a linear approximation for the small variations of $\Gamma(E)$ and $\Delta(E)$ :

$$
\frac{1}{2} \Gamma(E) \approx \frac{1}{2} \hat{\Gamma}+s(E-\hat{E}),
$$

and

$$
\Delta(E) \approx \hat{\Delta}+\tilde{s}(E-\hat{E}),
$$

where $\hat{E}$ is given by $\hat{E}=E_{0}+\Delta(\hat{E}), \hat{\Gamma}=\Gamma(\hat{E}), \hat{\Delta}=\Delta(\hat{E})$, and the slopes $s$ and $\tilde{s}$ are expected to be small. The resulting denominator in Eq. (9) is a quadratic in $(E-\hat{E})$. Its form may be simplified by completing its square, i.e., by using the relation $A x^{2}-2 B x+C=A[x-(B / A)]^{2}+\left[C-\left(B^{2} / A\right)\right]$. In terms of a dimensionless parameter $e$, linear in energy, and defined by

$$
e \equiv \frac{E-\left\{\hat{E}-\frac{1}{2} \hat{\Gamma} s /\left[(1-\tilde{s})^{2}+s^{2}\right]\right\}}{\frac{1}{2} \hat{\Gamma}(1-\tilde{s}) /\left[(1-\tilde{s})^{2}+s^{2}\right]},
$$

Eq. (9) may be rewritten, if $|\tilde{s}| \ll 1$, as

$$
\sigma \approx \frac{\sigma_{b}}{\pi} \frac{1}{\frac{1}{2} \hat{\Gamma}} \frac{1+s e}{1+e^{2}} .
$$

The profile defined by Eq. (13) is, for $s^{2} \ll 1$, a nearLorentzian, slightly asymmetric peak with an asymmetry (high-energy minus low-energy half widths at half maxima divided by the FWHM) very close to $s / 2$.

Under similar conditions of approximation, it is also of interest to consider the effect of energy-dependent parameters on the Fano line shape for predissociation by optically active continua. In the case of weak coupling, where the principal-value terms in the numerator of Eq. (7) may be neglected in comparison with the discrete transition moment, it follows from Eqs. (3), (7), and (8) that

$$
q^{2}(E) \sigma_{d}(E) \approx \frac{\sigma_{b}}{\pi} \frac{1}{\frac{1}{2} \Gamma(E)} .
$$

Substituting Eq. (14) into Eq. (1), if $(\epsilon / q)^{2} \ll 1$ we may write

$$
\sigma \approx \frac{\sigma_{b}}{\pi} \frac{1}{\frac{1}{2} \Gamma} \frac{1+(2 / q) \epsilon}{1+\epsilon^{2}}+\left(\sigma_{c}-\sigma_{d}\right) .
$$

Taking account of the definition of $\epsilon$ in Eq. (2), Eq. (15) may be simplified in the manner described above for Eq. (9) in the case of optically inactive continua. In addition to the energy dependences described by Eqs. (10) and (11) for $\Gamma$ and $\Delta$, respectively, $1 / q, \sigma_{d}$, and $\sigma_{c}$ are assumed to have weak, linear dependences on energy. If $|\tilde{s}| \ll 1$ and $|s / q| \ll 1$, it follows that

$$
\sigma \approx \frac{\sigma_{b}}{\pi} \frac{1}{\frac{1}{2} \hat{\Gamma}} \frac{1+(s+2 / \hat{q}) e}{1+e^{2}}+\left(\hat{\sigma}_{c}-\hat{\sigma}_{d}\right),
$$

where $\hat{q}=q(\hat{E}), \hat{\sigma}_{c}=\sigma_{c}(\hat{E}), \hat{\sigma}_{d}=\sigma_{d}(\hat{E})$, and the energy dependence of the $\left(\sigma_{c}-\sigma_{d}\right)$ background term has been neglected [17]. Thus, as in the case of Eq. (13) for optically inactive continua, it is the energy dependence of the width function that provides the major additional contribution to the line-shape asymmetry [18], which is very close to $(s / 2$ $+1 / \hat{q}$ ) for Eq. (16).

To within a constant background cross-section term, the line shapes defined by Eqs. (13) and (16) have the same form, which we will call the pseudo-Fano profile, and may be compared with the near-Lorentzian Fano profile defined by the constant-parameter version of Eq. (15). Thus, narrow, isolated predissociation resonance peaks for both optically inactive and optically active continua have, to a very good approximation, the form of a constant-parameter Fano profile with a large effective profile index $q^{\text {eff }}$ given by

$$
\frac{1}{q^{\mathrm{eff}}} \approx \frac{1}{\hat{q}}+\frac{1}{{ }^{(1)} q^{\mathrm{eff}}},
$$

where

$$
\frac{1}{{ }^{(1)} q^{\mathrm{eff}}} \approx \frac{s}{2},
$$

and $s$ is half the slope of the width function at the absorption peak. In Eq. (17), $1 /{ }^{(1)} q^{\text {eff }}$ represents the contribution to the inverse effective profile index caused specifically by energy dependences of the line-shape parameters, and is equal to its value in the case of predissociation by optically inactive continua $(1 / \hat{q}=0)$. 


\section{Interacting resonances}

The effects on line-shape asymmetry of interactions between resonances are revealed most transparently by considering the case of decay to a single continuum. That case has been addressed specifically by Fano [1] and Mies [3]. If the continuum is optically inactive, the photodissociation cross section may be written in the form [19]

$$
\sigma \approx \frac{\left[\sum_{v}\left(t_{v} / \pi V_{v}\right)\left(1 / \epsilon_{v}\right)\right]^{2}}{1+\left[\sum_{v}\left(1 / \epsilon_{v}\right)\right]^{2}}
$$

where the subscript $v$ indicates the vibrational quantum number of the dissociating state, $t_{v}=\left\langle\phi_{v}|\tau| \phi_{i}\right\rangle$ is the real, energy-independent transition amplitude to that state, and the energy parameters $\epsilon_{v}=2\left(E-E_{v}\right) / \Gamma_{v}$ [20]. We emphasise here that, in the NIRA, where there are rather weak couplings between the bound vibrational states and the continuum, it is a justifiable approximation to use the diabatic, or unshifted wave functions [1,3] in evaluating the relevant matrix elements in Eq. (19).

Equation (19) may be simplified further if the NIRA is applicable. In this case, the sums in its numerator and denominator may be split into two terms: an energy-dependent term associated with the main resonance $v$, and an energyindependent term associated with the sum over all other resonances $v^{\prime}$, since, for energies in the region of the main resonance, $1 / \epsilon_{v} \gg 1 / \epsilon_{v^{\prime}} \approx$ const. Using this approximation, and completing the square on the denominator, as in Sec. II B, we may write

$$
\sigma \approx \frac{\sigma_{b v}}{\pi} \frac{1}{\frac{1}{2} \Gamma_{v}} \frac{\left\{1+\left[\sum_{v^{\prime} \neq v}\left(t_{v^{\prime}} / t_{v}\right)\left(V_{v} / V_{v^{\prime}}\right)\left(1 / \epsilon_{v^{\prime}}\right)\right] \epsilon_{v}^{\prime}\right\}^{2}}{1+\epsilon_{v}^{\prime 2}}
$$

where $\sigma_{b v}=t_{v}^{2}$ is the integrated photoabsorption cross section to the dissociating state with vibrational quantum number $v$, the shifted energy parameter $\epsilon_{v}^{\prime}=\epsilon_{v}+\Sigma_{v^{\prime} \neq v}\left(1 / \epsilon_{v^{\prime}}\right)$, and all bound-continuum matrix elements are to be evaluated at $E=E_{v}$. Thus, in the NIRA, the line shape for predissociation by a single, optically inactive continuum, which includes the effects of indirect interactions with other resonances via the continuum, has the form of a Fano profile with an inverse effective profile index given by the sum in square brackets in the numerator of Eq. (20).

This result may be generalized to the case of weak predissociation by several continua, some of which may be optically active. Starting with the matrix equation for the partial photodissociation amplitudes [Eq. (35) of Ref. [3]], and using similar approximations to those above, we find

$$
\begin{aligned}
\sigma \approx & \frac{\sigma_{b v}}{\pi} \frac{1}{\frac{1}{2} \Gamma_{v}} \frac{1+\left(2 / q_{v}^{\mathrm{eff}}\right) \epsilon_{v}^{\prime \prime}}{1+\epsilon_{v}^{\prime \prime 2}}+\left(\sigma_{c}-\sigma_{d}\right) \\
& +2 \sum_{v^{\prime} \neq v} \frac{1}{\epsilon_{v^{\prime}}} \frac{1}{q_{v^{\prime}}} \frac{\sigma_{b v^{\prime}}}{(\pi / 2) \Gamma_{v^{\prime}}}
\end{aligned}
$$

where

$$
\begin{gathered}
\epsilon_{v}^{\prime \prime} \equiv \epsilon_{v}+\sum_{v^{\prime} \neq v} \frac{O_{v v^{\prime}}^{2}\left(E_{v}\right)}{\epsilon_{v^{\prime}}}, \\
\frac{1}{q_{v}^{\text {eff }}} \approx \frac{1}{q_{v}}+\frac{1}{{ }^{(2)} q_{v}^{\text {eff }}},
\end{gathered}
$$

and

$$
\begin{aligned}
\frac{1}{{ }^{(2)} q_{v}^{\mathrm{eff}}} & \approx \sum_{\alpha} \sum_{v^{\prime} \neq v} \frac{t_{v^{\prime}}}{t_{v}} \frac{V_{v^{\prime} \alpha}\left(E_{v}\right)}{V_{v \alpha}\left(E_{v}\right)} \frac{\frac{1}{2} \Gamma_{v \alpha}\left(E_{v}\right)}{E_{v}-E_{v^{\prime}}} \\
& =\pi \sum_{\alpha} \sum_{v^{\prime} \neq v} \frac{t_{v^{\prime}}}{t_{v}} \frac{V_{v \alpha}\left(E_{v}\right) V_{v^{\prime} \alpha}\left(E_{v}\right)}{E_{v}-E_{v^{\prime}}} \\
& =\frac{1}{2} \sum_{v^{\prime} \neq v} \frac{t_{v^{\prime}}}{t_{v}} \frac{\left[\Gamma_{v}\left(E_{v}\right) \Gamma_{v^{\prime}}\left(E_{v}\right)\right]^{1 / 2}}{E_{v}-E_{v^{\prime}}} O_{v v^{\prime}}\left(E_{v}\right)
\end{aligned}
$$

In Eqs. (22) and (24), the $O_{v v^{\prime}}\left(E_{v}\right)$ are elements of the overlap matrix introduced by Mies [3]. The nearly constant final term on the right-hand side (RHS) of Eq. (21), by comparison with the first, can be seen to represent the sum of the wings of the other resonances.

Thus, neglecting constant background terms, the NIRA line shape for predissociation by multiple optically active continua has the form of a pseudo-Fano profile. The effect of interactions between the resonances via the continua, as in the case of predissociation by a single, optically inactive continuum, is to modify the effective line-shape asymmetry, this contribution to the inverse effective profile index being given by Eq. (24). If the effects of energy-dependent parameters, as described in Sec. II B, are included for the main resonance, then the total inverse effective profile index is given by

$$
\frac{1}{q_{v}^{\text {eff }}} \approx \frac{1}{\hat{q}_{v}}+\frac{1}{{ }^{(1)} q_{v}^{\text {eff }}}+\frac{1}{{ }^{(2)} q_{v}^{\text {eff }}}
$$

\section{Sum rules}

A number of sum rules follow from Eq. (4) for noninteracting continua in the IRA. The width-function sum rule

$$
\Gamma(E)=\sum_{\alpha} \Gamma_{\alpha}(E)
$$




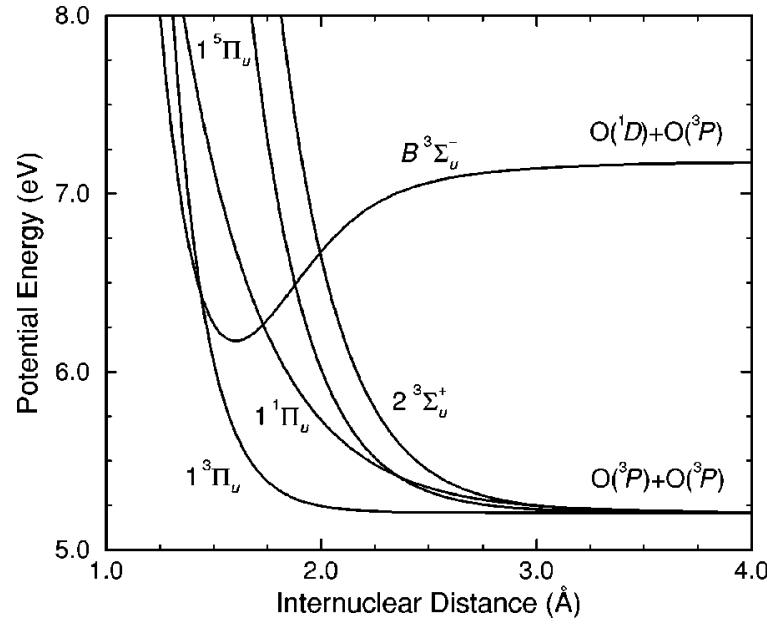

FIG. 1. Model potential-energy curves for states involved in the $B{ }^{3} \Sigma_{u}^{-}$-state predissociation. The energy scale is referred to the minimum in the ground-state $X^{3} \Sigma_{g}^{-}$potential.

where

$$
\Gamma_{\alpha}(E) \equiv 2 \pi V_{\alpha}^{2}(E)
$$

is the width function for dissociation into continuum $\alpha$ only, follows from Eqs. (3) and (4). If the resonance is so narrow

TABLE I. Magnitudes of percentage differences between CSEcalculated $B^{3} \Sigma_{u}^{-} \leftarrow X^{3} \Sigma_{g}^{-}(v, 0)$ line profiles (for optically inactive continua) and fitted Fano and Lorentzian profiles, respectively, as a function of the distance from the peak, in units of FWHM $(\Gamma)$.

\begin{tabular}{|c|c|c|c|c|c|c|}
\hline \multirow[b]{2}{*}{$v$} & \multicolumn{3}{|c|}{ Fano profile fit $^{\mathrm{a}}$} & \multicolumn{3}{|c|}{ Lorentzian fit } \\
\hline & $\pm 1 \Gamma$ & $\pm 10 \Gamma$ & $\pm 30 \Gamma$ & $\pm 1 \Gamma$ & $\pm 10 \Gamma$ & $\pm 30 \Gamma$ \\
\hline 0 & $5.8 \times 10^{-5}$ & 0.07 & 0.7 & 0.05 & 0.8 & 2.4 \\
\hline 1 & $2.7 \times 10^{-4}$ & 0.32 & 2.8 & 0.16 & 2.5 & 7.6 \\
\hline 2 & $1.5 \times 10^{-5}$ & 0.01 & 0.1 & 0.21 & 3.4 & 10.2 \\
\hline 3 & $2.4 \times 10^{-4}$ & 0.30 & 2.7 & 0.28 & 4.6 & 13.8 \\
\hline 4 & $1.0 \times 10^{-3}$ & 1.17 & 10.2 & 0.54 & 8.6 & 24.5 \\
\hline 5 & $9.6 \times 10^{-5}$ & 0.11 & 1.1 & 0.38 & 6.1 & 18.3 \\
\hline 6 & $2.5 \times 10^{-4}$ & 0.28 & 2.5 & 0.35 & 5.7 & 17.0 \\
\hline 7 & $1.2 \times 10^{-4}$ & 0.14 & 1.2 & 0.31 & 5.1 & 16.3 \\
\hline 8 & $5.3 \times 10^{-5}$ & 0.06 & 0.6 & 0.42 & 6.8 & 21.1 \\
\hline 9 & $1.5 \times 10^{-4}$ & 0.17 & 1.6 & 0.18 & 2.9 & 8.5 \\
\hline 10 & $2.7 \times 10^{-5}$ & 0.03 & 0.3 & 0.14 & 2.3 & 7.0 \\
\hline 11 & $1.2 \times 10^{-4}$ & 0.14 & 1.3 & 0.30 & 4.8 & 14.8 \\
\hline 12 & $2.0 \times 10^{-5}$ & 0.02 & 0.2 & 0.60 & 9.7 & 30.4 \\
\hline 13 & $4.5 \times 10^{-5}$ & 0.05 & 0.5 & 0.18 & 2.9 & 8.8 \\
\hline 14 & $8.2 \times 10^{-5}$ & 0.10 & 1.0 & 0.27 & 4.5 & 13.3 \\
\hline 15 & $1.5 \times 10^{-5}$ & 0.03 & 0.3 & 0.39 & 6.3 & 19.0 \\
\hline 16 & $8.5 \times 10^{-5}$ & 0.10 & 0.9 & 0.26 & 4.2 & 12.8 \\
\hline 17 & $1.4 \times 10^{-4}$ & 0.17 & 1.5 & 0.07 & 1.2 & 3.7 \\
\hline 18 & $1.5 \times 10^{-4}$ & 0.18 & 1.6 & 0.08 & 1.3 & 3.8 \\
\hline 19 & $1.3 \times 10^{-4}$ & 0.16 & 1.4 & 0.18 & 2.9 & 8.7 \\
\hline 20 & $1.1 \times 10^{-4}$ & 0.13 & 1.3 & 0.22 & 3.6 & 11.0 \\
\hline 21 & $8.9 \times 10^{-5}$ & 0.13 & 1.2 & 0.22 & 3.5 & 10.7 \\
\hline
\end{tabular}

$\overline{{ }^{\mathrm{a}} \mathrm{CSE} \text { profiles fitted to constant-parameter version of Eq. (1) with a }}$ zero background term $\left(\sigma_{c}-\sigma_{d}\right)$.
TABLE II. Magnitudes of percentage differences between CSEcalculated $B^{3} \Sigma_{u}^{-} \leftarrow X^{3} \Sigma_{g}^{-}(v, 0)$ line profiles for an optically active ${ }^{3} \Pi_{u}$ continuum and fitted Fano profiles, as a function of the distance from the peak, in units of FWHM $(\Gamma)$. The CSE profiles have been fitted to the constant-parameter version of Eq. (1).

\begin{tabular}{lcccccc}
\hline \hline & \multicolumn{2}{c}{$\tau_{\alpha={ }^{3} \Pi_{u}}=0.3$ a.u. } & \multicolumn{2}{c}{$\tau_{\alpha={ }^{3} \Pi_{u}}=1.0$ a.u. } \\
$v$ & $\pm 1 \Gamma$ & $\pm 10 \Gamma$ & $\pm 30 \Gamma$ & $\pm 1 \Gamma$ & $\pm 10 \Gamma$ & $\pm 30 \Gamma$ \\
\hline 0 & $1.9 \times 10^{-5}$ & 0.006 & 0.16 & $2.8 \times 10^{-4}$ & 0.074 & 0.59 \\
1 & $2.9 \times 10^{-4}$ & 0.094 & 1.92 & $4.5 \times 10^{-3}$ & 0.841 & 4.71 \\
2 & $1.8 \times 10^{-5}$ & 0.006 & 0.16 & $3.2 \times 10^{-4}$ & 0.106 & 1.55 \\
3 & $1.4 \times 10^{-4}$ & 0.046 & 1.25 & $2.2 \times 10^{-3}$ & 0.628 & 5.54 \\
4 & $4.3 \times 10^{-4}$ & 0.145 & 3.35 & $5.6 \times 10^{-3}$ & 1.397 & 10.19 \\
5 & $9.4 \times 10^{-6}$ & 0.003 & 0.07 & $1.3 \times 10^{-3}$ & 0.373 & 4.22 \\
6 & $4.2 \times 10^{-5}$ & 0.019 & 0.55 & $6.4 \times 10^{-4}$ & 0.218 & 3.30 \\
7 & $4.8 \times 10^{-5}$ & 0.021 & 0.52 & $9.5 \times 10^{-4}$ & 0.276 & 3.33 \\
8 & $2.0 \times 10^{-5}$ & 0.006 & 0.19 & $4.1 \times 10^{-4}$ & 0.135 & 2.17 \\
9 & $8.9 \times 10^{-6}$ & 0.001 & 0.04 & $8.8 \times 10^{-5}$ & 0.030 & 0.71 \\
10 & $1.9 \times 10^{-5}$ & 0.006 & 0.17 & $1.2 \times 10^{-4}$ & 0.039 & 0.70 \\
11 & $5.6 \times 10^{-5}$ & 0.019 & 0.54 & $1.6 \times 10^{-4}$ & 0.050 & 0.80 \\
12 & $1.3 \times 10^{-5}$ & 0.004 & 0.13 & $2.4 \times 10^{-5}$ & 0.009 & 0.23 \\
13 & $8.8 \times 10^{-7}$ & 0.000 & 0.00 & $1.2 \times 10^{-6}$ & 0.000 & 0.01 \\
14 & $5.5 \times 10^{-6}$ & 0.000 & 0.00 & $5.9 \times 10^{-6}$ & 0.001 & 0.03 \\
15 & $1.3 \times 10^{-5}$ & 0.001 & 0.02 & $1.4 \times 10^{-5}$ & 0.004 & 0.09 \\
16 & $4.5 \times 10^{-6}$ & 0.001 & 0.03 & $6.5 \times 10^{-6}$ & 0.001 & 0.02 \\
17 & $1.9 \times 10^{-5}$ & 0.006 & 0.17 & $2.6 \times 10^{-5}$ & 0.008 & 0.14 \\
18 & $3.2 \times 10^{-5}$ & 0.011 & 0.29 & $5.1 \times 10^{-5}$ & 0.016 & 0.29 \\
19 & $3.9 \times 10^{-5}$ & 0.013 & 0.36 & $6.5 \times 10^{-5}$ & 0.021 & 0.38 \\
20 & $4.0 \times 10^{-5}$ & 0.014 & 0.38 & $7.1 \times 10^{-5}$ & 0.023 & 0.43 \\
21 & $3.5 \times 10^{-5}$ & 0.014 & 0.40 & $7.0 \times 10^{-5}$ & 0.025 & 0.48 \\
\hline \hline & & & & & & \\
\hline
\end{tabular}

that $\Gamma$ can be taken to be constant over its peak, then the well-known width sum rule $[1,3,15]$ follows. Similarly, from Eq. (6) for the shift function, the sum rule for $\Gamma(E)$ implies the following sum rule for the shift function:

$$
\Delta(E)=\sum_{\alpha} \Delta_{\alpha}(E)
$$

where

$$
\Delta_{\alpha}(E) \equiv \frac{1}{2 \pi} \mathrm{P} \int d E^{\prime} \frac{\Gamma_{\alpha}\left(E^{\prime}\right)}{E-E^{\prime}}
$$

is the shift function for dissociation into continuum $\alpha$ only. Again, if the resonance is narrow enough that $\Delta$ may be taken constant, the energy-shift sum rule [15] follows.

In the case of a Fano predissociation profile, where, by definition, the energy dependences of parameters over the peak may be neglected, the principal-value integrals in Eq. (7) are negligible, so that

$$
\frac{1}{q}=\sum_{\alpha} \frac{1}{q_{\alpha}}
$$

where 


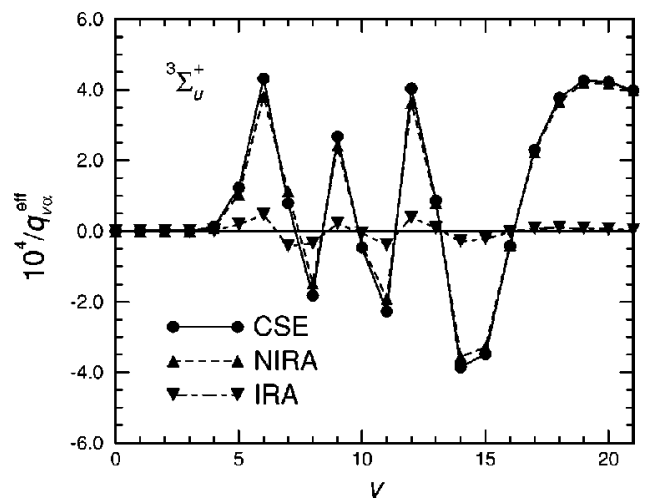

FIG. 2. Comparison between exact $B{ }^{3} \Sigma_{u}^{-} \leftarrow X^{3} \Sigma_{g}^{-}(v, 0)$ lineshape asymmetries $1 / q_{v \alpha}^{\text {eff }}$ for predissociation by a single, inactive $\alpha={ }^{3} \Sigma_{u}^{+}$continuum, calculated using the CSE, and perturbative estimates of the specific contributions due to interactions with other resonances $\left[1 /^{(2)} q_{v \alpha}^{\text {eff }}\right.$, Eq. (24), labeled NIRA], and due to the energy dependence of the width parameter $\left[1 / /^{(1)} q_{v \alpha}^{\text {eff }}\right.$, Eq. (18), labeled IRA].

$$
q_{\alpha} \equiv \frac{\left\langle\phi|\tau| \phi_{i}\right\rangle}{\pi V_{\alpha}\left\langle\mathcal{R}_{\alpha}\left|\tau_{\alpha}\right| \phi_{i}\right\rangle}
$$

is the profile index for dissociation to continuum $\alpha$ only. Thus, a Fano profile for an isolated state dissociating to several continua satisfies an inverse profile index sum rule.

We consider now the total inverse effective profile index defined by Eq. (25), which contains the effects of energydependent parameters and interacting resonances discussed in Secs. II B and II C, respectively, and introduce an effective index $q_{v \alpha}^{\text {eff }}$ for the corresponding profile for dissociation into continuum $\alpha$ only:

$$
\frac{1}{q_{v \alpha}^{\text {eff }}} \approx \frac{1}{\hat{q}_{v \alpha}}+\frac{1}{{ }^{(1)} q_{v \alpha}^{\text {eff }}}+\frac{1}{{ }^{(2)} q_{v \alpha}^{\text {eff }}}
$$

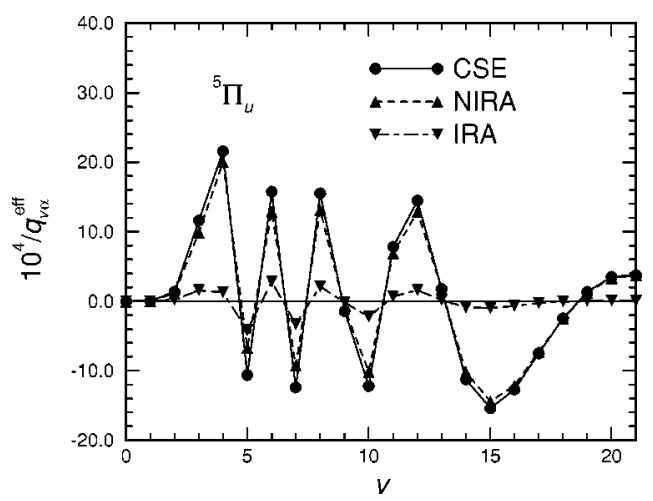

FIG. 3. Comparison between exact $B^{3} \Sigma_{u}^{-} \leftarrow X^{3} \Sigma_{g}^{-}(v, 0)$ lineshape asymmetries $1 / q_{v \alpha}^{\text {eff }}$ for predissociation by a single, inactive $\alpha={ }^{5} \Pi_{u}$ continuum, calculated using the CSE, and perturbative estimates of the specific contributions due to interactions with other resonances $\left[1 /{ }^{(2)} q_{v \alpha}^{\text {eff }}\right.$, Eq. (24), labeled NIRA], and due to the energy dependence of the width parameter $\left[1 /{ }^{(1)} q_{v \alpha}^{\text {eff }}\right.$, Eq. (18), labeled IRA].

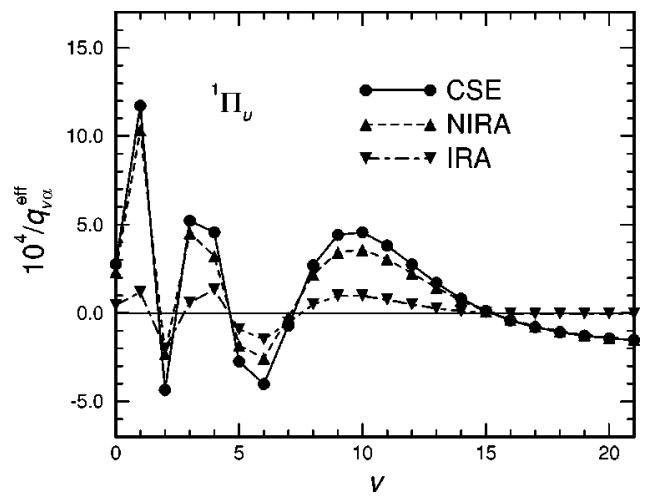

FIG. 4. Comparison between exact $B^{3} \Sigma_{u}^{-} \leftarrow X^{3} \Sigma_{g}^{-}(v, 0)$ lineshape asymmetries $1 / q_{v \alpha}^{\text {eff }}$ for predissociation by a single, inactive $\alpha={ }^{1} \Pi_{u}$ continuum, calculated using the CSE, and perturbative estimates of the specific contributions due to interactions with other resonances $\left[1 / /^{(2)} q_{v \alpha}^{\text {eff }}\right.$, Eq. (24), labeled NIRA], and due to the energy dependence of the width parameter $\left[1 / /^{(1)} q_{v \alpha}^{\text {eff }}\right.$, Eq. (18), labelled IRA].

From Eq. (30), it follows that the first term on the RHS of Eq. (32) obeys a sum rule on $\alpha$. From Eqs. (10) and (26), it follows that

$$
s_{v}=\sum_{\alpha} s_{v \alpha}
$$

where $s_{v \alpha}$ is the slope of $\frac{1}{2} \Gamma_{v \alpha}$. Equations (18) and (33) imply that the second term on the RHS of Eq. (32) obeys a similar sum rule. Finally, the form of Eq. (24) indicates that the third term on the RHS of Eq. (32) also obeys a sum rule. Therefore, we may write

$$
\frac{1}{q_{v}^{\mathrm{eff}}} \approx \sum_{\alpha} \frac{1}{q_{v \alpha}^{\mathrm{eff}}}
$$

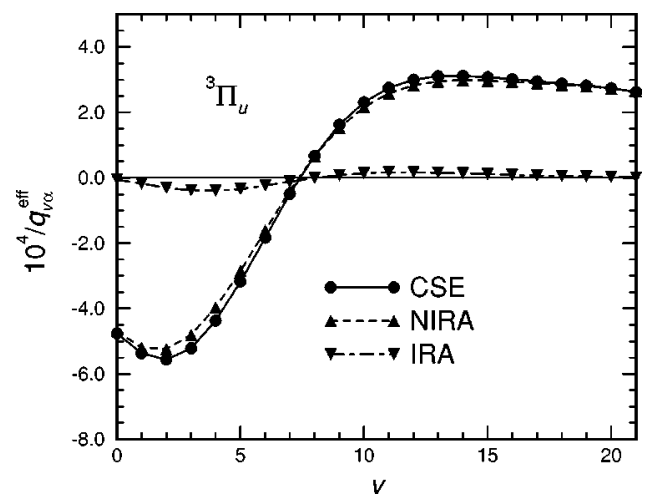

FIG. 5. Comparison between exact $B^{3} \Sigma_{u}^{-} \leftarrow X^{3} \Sigma_{g}^{-}(v, 0)$ lineshape asymmetries $1 / q_{v \alpha}^{\text {eff }}$ for predissociation by a single, inactive $\alpha={ }^{3} \Pi_{u}$ continuum, calculated using the CSE, and perturbative estimates of the specific contributions due to interactions with other resonances $\left[1 / /^{(2)} q_{v \alpha \alpha}^{\text {eff }}\right.$, Eq. (24), labeled NIRA], and due to the energy dependence of the width parameter $\left[1 /{ }^{(1)} q_{v \alpha}^{\text {eff }}\right.$, Eq. (18), labeled IRA]. 
TABLE III. Comparison of calculated single-channel and multichannel predissociation line widths for $\mathrm{O}_{2}\left[B^{3} \Sigma_{u}^{-}(v)\right]$, in units of $\mathrm{cm}^{-1}$ FWHM. The linewidths are from zero-background Fano-profile fits to CSE line shapes for SR predissociation by optically inactive continua.

\begin{tabular}{lrlllllr}
\hline \hline$v$ & $\alpha={ }^{3} \Pi_{u}{ }^{\mathrm{a}}$ & $\alpha={ }^{5} \Pi_{u}{ }^{\mathrm{a}}$ & $\alpha={ }^{1} \Pi_{u}{ }^{\mathrm{a}}$ & $\alpha={ }^{3} \Sigma_{u}{ }^{\mathrm{a}}{ }^{\mathrm{a}}$ & $\Sigma_{\alpha} \Gamma_{v \alpha}$ & $\Gamma_{v}{ }^{\mathrm{b}}$ & $\%_{\text {Diff. }}{ }^{\mathrm{a}}$ \\
\hline 0 & 0.01715 & 0.00002 & 0.11670 & 0.00000 & 0.13386 & 0.13395 & 0.07 \\
1 & 0.05994 & 0.00216 & 1.06302 & 0.00000 & 1.12512 & 1.12552 & 0.04 \\
2 & 0.12130 & 0.07691 & 0.28066 & 0.00000 & 0.47887 & 0.47798 & -0.18 \\
3 & 0.18587 & 0.89974 & 0.65800 & 0.00022 & 1.74383 & 1.74362 & -0.01 \\
4 & 0.23901 & 3.14506 & 0.10205 & 0.00680 & 3.49291 & 3.49455 & 0.05 \\
5 & 0.27191 & 1.67408 & 0.04123 & 0.09093 & 2.07814 & 2.07692 & -0.06 \\
6 & 0.28088 & 0.62063 & 0.25285 & 0.48457 & 1.63892 & 1.63948 & 0.03 \\
7 & 0.26996 & 0.99308 & 0.35145 & 0.73624 & 2.35073 & 2.35253 & 0.08 \\
8 & 0.24349 & 1.07505 & 0.30502 & 0.03248 & 1.65604 & 1.65501 & -0.06 \\
9 & 0.20783 & 0.00288 & 0.20442 & 0.39517 & 0.81030 & 0.80958 & -0.09 \\
10 & 0.17037 & 0.74388 & 0.11471 & 0.00118 & 1.03013 & 1.03038 & 0.02 \\
11 & 0.13326 & 0.85503 & 0.05436 & 0.25194 & 1.29458 & 1.29521 & 0.05 \\
12 & 0.10155 & 0.27439 & 0.02170 & 0.15171 & 0.54935 & 0.54791 & -0.26 \\
13 & 0.07492 & 0.00222 & 0.00668 & 0.00210 & 0.08593 & 0.08533 & -0.70 \\
14 & 0.05399 & 0.08602 & 0.00124 & 0.04542 & 0.18667 & 0.18685 & 0.10 \\
15 & 0.03839 & 0.21477 & 0.00002 & 0.09868 & 0.35187 & 0.35229 & 0.12 \\
16 & 0.02705 & 0.25842 & 0.00020 & 0.09456 & 0.38023 & 0.38067 & 0.12 \\
17 & 0.01898 & 0.23425 & 0.00059 & 0.06550 & 0.31933 & 0.31958 & 0.08 \\
18 & 0.01322 & 0.18391 & 0.00082 & 0.03864 & 0.23659 & 0.23665 & 0.02 \\
19 & 0.00899 & 0.13134 & 0.00084 & 0.02086 & 0.16203 & 0.16199 & -0.03 \\
20 & 0.00570 & 0.08464 & 0.00069 & 0.01050 & 0.10153 & 0.10147 & -0.06 \\
21 & 0.00300 & 0.04458 & 0.00042 & 0.00461 & 0.05260 & 0.05256 & -0.09 \\
\hline \hline
\end{tabular}

${ }^{\mathrm{a}}$ From CSE calculations with indicated single dissociative channel.

${ }^{\mathrm{b}}$ From CSE calculations with four simultaneous dissociative channels.

${ }^{\mathrm{c}} 100\left(\Gamma_{v}-\Sigma_{\alpha} \Gamma_{v \alpha}\right) / \Gamma_{v}$.

similar to the result Eq. (30) for the Fano profile. As the asymmetry of a profile is very close to the inverse of its effective profile index, the sum rule Eq. (34) can be regarded as an "asymmetry sum rule." Such sum rules do not appear to have been reported previously.

\section{COMPUTATION}

\section{A. Predissociation model}

Our description of predissociation in the SR $\left[B^{3} \Sigma_{u}^{-}\right.$ $\left.\leftarrow X^{3} \Sigma_{g}^{-}(v, 0)\right]$ bands of $\mathrm{O}_{2}$ is based on the model of Julienne and Krauss [21] and Julienne [22], whereby the bound $B^{3} \Sigma_{u}^{-}$state couples, principally by spin-orbit interaction, to four repulsive states, $1^{3} \Pi_{u}, 1^{1} \Pi_{u}, 1^{5} \Pi_{u}$, and $2^{3} \Sigma_{u}^{+}$. The particular implementation of that model used here is that of Lewis et al. [12]. Briefly, the $X$ - and $B$-state Rydberg-KleinRees potential-energy curves and electronic transition moments of Ref. [23], and the exponential repulsive potentials and $R$-independent spin-orbit couplings of Ref. [24] were employed. The model potential-energy curves are shown in Fig. 1. Calculations were performed only for the $F_{2}$ levels of ${ }^{16} \mathrm{O}_{2}(\Omega=1, J=N, f$ parity [25]) in the rotationless case, so rotational coupling between the $B^{3} \Sigma_{u}^{-}$and $1^{3} \Pi_{u}$ states [21] was not considered [26].

Since the spin-orbit couplings are all less than $100 \mathrm{~cm}^{-1}$ [24], and the corresponding SR predissociation line widths are less than $4 \mathrm{~cm}^{-1}$ [27], small compared with the vibrational spacing, the NIRA is satisfied for this system. Since the crossing points of the various repulsive curves with the $B$-state potential are well separated (see Fig. 1), and since they have only weak mutual electronic interactions, the condition of noninteracting continua is satisfied. Finally, transitions from the $X^{3} \Sigma_{g}^{-}$state to the continua are electric-dipole forbidden, except for that to the $1^{3} \Pi_{u}$ state. The electronic transition moment for the $1^{3} \Pi_{u} \leftarrow X^{3} \Sigma_{g}^{-}$transition is known to be very small $[28,29]$ and there is very poor FranckCondon overlap with the ground state in the region of the SR bands. Therefore, the condition of optically inactive continua is effectively satisfied. Thus, the SR sytem of $\mathrm{O}_{2}$, of such importance to the photochemistry of the terrestrial atmosphere, provides a convenient test bed for numerical investigation of the validity of the main findings of Sec. II.

The results to be discussed in Sec. III B were obtained from various perturbative and CSE calculations. Uncoupled radial wave functions for the diabatic potentials employed in the perturbative CI calculations were obtained using the renormalized Numerov method of Johnson [30]. Multichannel SR photodissociation cross sections, calculated using the diabatic CSE formalism described in Ref. [31], with the predissociation model outlined above, were used to obtain numerical resonance line shapes for the rotationless $B \leftarrow X(0,0)$ to $(21,0)$ transitions for comparison with the theory of Sec. 
TABLE IV. Comparison of calculated single-channel and multichannel energy shifts for $\mathrm{O}_{2}\left[B^{3} \Sigma_{u}^{-}(v)\right]$, in $\mathrm{cm}^{-1}$. The energy shifts are derived from line centers obtained from zero-background Fano-profile fits to CSE line shapes for SR predissociation by optically inactive continua.

\begin{tabular}{lrrrrrrr}
\hline \hline$v$ & $\alpha={ }^{3} \Pi_{u}{ }^{\mathrm{a}}$ & \multicolumn{1}{c}{$\alpha={ }^{5} \Pi_{u}{ }^{\mathrm{a}}$} & \multicolumn{1}{c}{$\alpha={ }^{1} \Pi_{u}{ }^{\mathrm{a}}$} & \multicolumn{1}{c}{$\alpha={ }^{3} \Sigma_{u}{ }^{\mathrm{a}}$} & $\Sigma_{\alpha} \Delta_{v \alpha}$ & \multicolumn{1}{c}{$\Delta_{v}{ }^{\mathrm{b}}$} & $\%$ Diff. $^{\mathrm{c}}$ \\
\hline 0 & 0.17311 & -0.29844 & -0.35838 & $-0.04039^{\mathrm{d}}$ & -0.52411 & -0.52369 & -0.08 \\
1 & 0.18905 & -0.39748 & -0.29835 & $-0.04789^{\mathrm{e}}$ & -0.55467 & -0.55447 & -0.04 \\
2 & 0.19415 & -0.61549 & 0.24704 & -0.05892 & -0.23323 & -0.23344 & 0.09 \\
3 & 0.18501 & -1.01597 & -0.03081 & -0.07668 & -0.93846 & -0.93845 & 0.00 \\
4 & 0.16332 & -0.55113 & -0.06923 & -0.11178 & -0.56882 & -0.56879 & -0.01 \\
5 & 0.13376 & 0.95636 & 0.10730 & -0.18692 & 1.01051 & 1.01094 & 0.04 \\
6 & 0.10174 & -0.18218 & 0.14273 & -0.21896 & -0.15666 & -0.15740 & 0.47 \\
7 & 0.07226 & 0.72951 & 0.07581 & 0.09541 & 0.97300 & 0.97315 & 0.02 \\
8 & 0.04817 & -0.01221 & 0.01086 & 0.14475 & 0.19156 & 0.19133 & -0.12 \\
9 & 0.03103 & 0.34433 & -0.01581 & 0.02881 & 0.38836 & 0.38844 & 0.02 \\
10 & 0.02052 & 0.56384 & -0.01292 & 0.09387 & 0.66531 & 0.66555 & 0.04 \\
11 & 0.01598 & 0.22566 & 0.00456 & 0.13163 & 0.37783 & 0.37739 & -0.12 \\
12 & 0.01545 & 0.14997 & 0.02356 & 0.03088 & 0.21985 & 0.21960 & -0.11 \\
13 & 0.01773 & 0.32113 & 0.03970 & 0.07885 & 0.45741 & 0.45789 & 0.10 \\
14 & 0.02140 & 0.44196 & 0.05121 & 0.12537 & 0.63993 & 0.64027 & 0.05 \\
15 & 0.02536 & 0.45337 & 0.05846 & 0.11512 & 0.65231 & 0.65247 & 0.02 \\
16 & 0.02905 & 0.41824 & 0.06262 & 0.09292 & 0.60283 & 0.60281 & 0.00 \\
17 & 0.03218 & 0.38406 & 0.06474 & 0.08190 & 0.56289 & 0.56276 & -0.02 \\
18 & 0.03473 & 0.36412 & 0.06567 & 0.08066 & 0.54517 & 0.54503 & -0.03 \\
19 & 0.03680 & 0.35659 & 0.06594 & 0.08357 & 0.54291 & 0.54281 & -0.09 \\
20 & 0.03856 & 0.35683 & 0.06586 & 0.08737 & 0.54862 & 0.54855 & -0.01 \\
21 & 0.04003 & 0.36066 & 0.06551 & 0.09071 & 0.55691 & 0.55687 & -0.01 \\
\hline \hline
\end{tabular}

${ }^{\mathrm{a}}$ From CSE calculations with indicated single dissociative channel.

${ }^{\mathrm{b}}$ From CSE calculations with four simultaneous dissociative channels.

${ }^{\mathrm{c}} 100\left(\Delta_{v}-\Sigma_{\alpha} \Delta_{v \alpha}\right) / \Delta_{v}$.

${ }^{\mathrm{d}}$ Fitting region $\sim 500 \mathrm{FWHM}$.

${ }^{\mathrm{e}}$ Fitting region $\sim 70 \mathrm{FWHM}$.

II. In addition, in order to validate the sum rules, singlechannel calculations, i.e., solutions of the CSE for the cases of each dissociative channel $\alpha$ of the four, $\alpha=1{ }^{5} \Pi_{u}, 1^{3} \Pi_{u}$, $1^{1} \Pi_{u}$, and $2^{3} \Sigma_{u}^{+}$, coupled to the $B^{3} \Sigma_{u}^{-}$state one at a time, were performed using the same predissociation model. Finally, indicative calculations were performed for the case of optically active continua by artificially assigning nonzero values to the $1^{3} \Pi_{u} \leftarrow X^{3} \Sigma_{g}^{-}$electronic transition moment.

\section{B. Results and discussion}

\section{Line shapes}

In the case of optically inactive continua, each SR resonance cross section computed using the CSE method and the full (four dissociative channels) predissociation model described in Sec. III A was least-squares fitted [32] both to a zero-background Fano profile [33] and to a Lorentzian profile. Where possible, the range of energies for the fit was chosen so that the cross section at the left and right end points was down by a factor of 100 compared with the peak cross section. This corresponds to a range of energies of about 10 FWHM, with the peak approximately centered. Within this range, the fit parameters were quite insensitive to the exact width of the energy interval.
Table I shows a comparison of the resulting best Lorentzian and Fano-profile fits to the $(0,0)$ through $(21,0)$ rotationless SR absorption peaks. These results confirm that the Fano profile provides a much better description of the real predissociation line shape than does the Lorentzian. The largest discrepancy in the Fano-profile fits of Table I occurs for $v$ $=4$, the broadest resonance in the SR system. A detuning of \pm 30 FWHM from this resonance corresponds to $\pm 105 \mathrm{~cm}^{-1}$, or $17 \%$ of the mean vibrational spacing of $605 \mathrm{~cm}^{-1}$ [23]. Thus, the $10 \%$ difference between the calculated $(4,0)$ profile and the Fano fit for this detuning is likely to be related to the the beginnings of breakdown of the NIRA.

Comparable indicative calculations have also been performed for the case of optically active continua. Multichannel CSE cross sections for an active ${ }^{3} \Pi_{u}$ continuum were fitted to a constant-background Fano profile. The results, given in Table II for two values of the ${ }^{3} \Pi_{u} \leftarrow X^{3} \Sigma_{g}^{-}$electronic transition moment, show, as in the inactive-continuum case, that the predissociation line shape is well represented by a Fano profile. In order to retain this degree of agreement for values of the electronic transition moment significantly larger than 1 a.u., however, it is necessary to take into account the explicit energy dependence of the background continuum component. 
TABLE V. Comparison of calculated single-channel and multichannel effective Fano profile indices $q^{\text {eff }}$ for the $B^{3} \Sigma_{u}^{-} \leftarrow X^{3} \Sigma_{g}^{-}(v, 0)$ bands of $\mathrm{O}_{2}$ (for optically inactive continua), including verification of the $1 / q^{\text {eff }}$ sum rule. The effective profile indices are from zero-background Fano-profile fits to CSE line shapes.

\begin{tabular}{lrrrrrrr}
\hline \hline$v$ & $\alpha={ }^{3} \Pi_{u}{ }^{\mathrm{a}}$ & \multicolumn{1}{c}{$\alpha={ }^{5} \Pi_{u}{ }^{\mathrm{a}}$} & \multicolumn{1}{c}{$\alpha={ }^{1} \Pi_{u}{ }^{\mathrm{a}}$} & $\alpha={ }^{3} \Sigma_{u}{ }_{u}^{\mathrm{a}}$ & $1 / \Sigma_{\alpha}\left(1 / q_{v \alpha}^{\text {eff }}\right)$ & \multicolumn{1}{c}{$q_{v}^{\text {eff b }}$} & $\%$ Diff. $^{\mathrm{c}}$ \\
\hline 0 & -2100.5 & $1.1 \times 10^{7}$ & 3617.7 & & -5010.6 & -5016.0 & 0.11 \\
1 & -1862.2 & $2.0 \times 10^{5}$ & 852.9 & & 1561.5 & 1560.3 & -0.08 \\
2 & -1798.0 & 7301.2 & -2303.6 & $4.9 \times 10^{5}$ & -1174.7 & -1171.4 & -0.28 \\
3 & -1917.5 & 864.0 & 1918.8 & $2.3 \times 10^{6}$ & 864.0 & 864.8 & 0.10 \\
4 & -2287.6 & 464.2 & 2191.7 & 85990.0 & 457.7 & 457.4 & -0.07 \\
5 & -3143.4 & -941.2 & -3653.6 & 8141.6 & -653.0 & -652.0 & -0.15 \\
6 & -5453.3 & 635.6 & -2492.5 & 2315.2 & 703.8 & 702.9 & -0.13 \\
7 & -20093.0 & -806.1 & -13800.0 & 12586.0 & -779.3 & -780.8 & 0.20 \\
8 & 14851.0 & 645.2 & 3714.6 & -5458.1 & 587.1 & 586.2 & -0.16 \\
9 & 6159.9 & -7050.7 & 2259.4 & 3737.2 & 1368.6 & 1368.8 & 0.02 \\
10 & 4337.8 & -818.9 & 2189.4 & -21660.0 & -1723.9 & -1719.7 & -0.25 \\
11 & 3632.2 & 1278.7 & 2620.7 & -4394.2 & 825.5 & 823.5 & -0.25 \\
12 & 3331.2 & 691.4 & 3636.5 & 2481.5 & 412.5 & 412.2 & -0.06 \\
13 & 3216.2 & 5673.4 & 5872.2 & 11602.0 & 1344.7 & 1352.7 & 0.60 \\
14 & 3206.9 & -889.6 & 12262.0 & -2595.1 & -896.0 & -892.6 & -0.38 \\
15 & 3251.4 & -648.5 & 84582.0 & -2866.3 & -636.3 & -635.3 & -0.15 \\
16 & 3316.0 & -785.4 & -24571.0 & -23885.0 & -948.6 & -948.9 & 0.03 \\
17 & 3389.8 & -1335.6 & -12587.0 & 4339.4 & -3303.4 & -3336.2 & 0.98 \\
18 & 3469.3 & -4075.7 & -9289.5 & 2652.8 & 3203.1 & 3159.7 & -1.37 \\
19 & 3547.1 & 7432.6 & -7825.0 & 2347.3 & 1399.2 & 1390.6 & -0.62 \\
20 & 3649.5 & 2873.6 & -7043.9 & 2369.8 & 1108.6 & 1103.6 & -0.46 \\
21 & 3811.9 & 2686.6 & -6594.6 & 2509.2 & 1134.5 & 1130.0 & -0.40 \\
\hline \hline
\end{tabular}

${ }^{\mathrm{a}}$ From CSE calculations with indicated single dissociative channel.

${ }^{\mathrm{b}}$ From CSE calculations with four simultaneous dissociative channels.

${ }^{\mathrm{c}} 100\left[q_{v}^{\text {eff }}-1 / \Sigma_{\alpha}\left(1 / q_{v \alpha}^{\text {eff }}\right)\right] / q_{v}^{\text {eff }}$.

\section{Asymmetry}

It is of interest to assess the relative importances of the two factors suggested in Sec. II to contribute to the effective asymmetry of the predissociation line shape $\left(\approx 1 / q^{\mathrm{eff}}\right)$. Therefore, a comprehensive study was performed for the case of SR predissociation by each single, inactive continuum.

First, the role of the width-parameter energy dependence discussed in Sec. II B was investigated by evaluating the energy-dependent matrix elements $V_{v \alpha}$ and estimating the corresponding contribution $1 /{ }^{(1)} q_{v \alpha}^{\text {eff }}$ using Eqs. (5) and (18), respectively. Second, in order to assess the role of the interactions between resonances described in Sec. II C, the perturbation sum in Eq. (24) was evaluated to give $1 /^{(2)} q_{v \alpha}^{\text {eff }}$ using the same couplings and transition moments employed in the CSE calculations, together with bound and energynormalized continuum wave functions appropriate to the relevant diabatic potential-energy curves. In order to evaluate $1 /^{(2)} q_{v \alpha}^{\text {eff }}$ correctly, it was necessary not only to perform the sum over all discrete levels of the $B$ state, but also to integrate over the $B$-state continuum. Finally, both of these contributions to $1 / q_{v \alpha}^{\text {eff }}$ were compared with values resulting from zero-background Fano-profile fits to the corresponding exact single-channel CSE calculations.

These results are shown in Figs. 2, 3, 4, and 5, for predissociation by the ${ }^{3} \Sigma_{u}^{+},{ }^{5} \Pi_{u},{ }^{1} \Pi_{u}$, and ${ }^{3} \Pi_{u}$ states, respec- tively. Inspection of these figures shows clearly that the lineshape asymmetry is dominated by the contribution arising from interactions between the resonance of interest and other resonances, with a smaller contribution arising from the energy dependence of the width parameter for the dominant resonance. The sums of these two contributions provide excellent descriptions of the exact CSE asymmetries for each curve crossing. Thus, perturbative CI calculations have provided significant insight into the causes of line-shape asymmetry that is not attainable from the CSE calculations directly.

It might be thought that the small degree of asymmetry involved in systems that are weakly coupled to optically inactive continua would be insufficient to be observable experimentally. This is not the case. The most asymmetric SR resonance occurs for $v^{\prime}=12$, with $q=+412.5$ (multichannel CSE calculation, see Sec. III B 3), implying a stronger blue wing. An asymmetry of this sense has been shown to occur in the experimental $79 \mathrm{~K}(12,0)$ SR-band cross section of Yoshino et al. [34] by Lewis et al. [12], the effect being clearly observable within 100 FWHM of the bandhead. Of course, at greater detunings, where the IRA and NIRA break down, the Fano profile becomes inapplicable. In the case of the $(12,0)$ resonance, CSE calculations [12] show that additional interference effects cause the blue wing to become significantly weaker than a Lorent- 
TABLE VI. Verification of the $1 / q^{\text {eff }}$ sum rule for the $B^{3} \Sigma_{u}^{-} \leftarrow X^{3} \Sigma_{g}^{-}(v, 0)$ bands of $\mathrm{O}_{2}$ in the case of an optically active ${ }^{3} \Pi_{u}$ continuum. The effective profile indices are from constant-background Fano-profile fits to CSE line shapes.

\begin{tabular}{|c|c|c|c|c|c|c|c|c|}
\hline \multirow[b]{2}{*}{$v$} & \multicolumn{4}{|c|}{$\tau_{\alpha={ }^{3} \Pi_{u}}=0.3$ a.u. } & \multicolumn{4}{|c|}{$\tau_{\alpha={ }^{3} \Pi_{u}}=1.0$ a.u. } \\
\hline & $\alpha={ }^{3} \Pi_{u}^{\mathrm{a}}$ & $1 / \Sigma_{\alpha}\left(1 / q_{v \alpha}^{\mathrm{eff}}\right)^{\mathrm{b}}$ & $q_{v}^{\mathrm{eff} \mathrm{c}}$ & $\%$ Diff. $^{\mathrm{d}}$ & $\alpha={ }^{3} \Pi_{u}{ }^{\mathrm{a}}$ & $1 / \Sigma_{\alpha}\left(1 / q_{v \alpha}^{\mathrm{eff}}\right)^{\mathrm{b}}$ & $q_{v}^{\text {eff c }}$ & $\%$ Diff. $^{\mathrm{d}}$ \\
\hline 0 & 311.2 & 286.5 & 286.5 & -0.01 & 84.2 & 82.3 & 82.3 & -0.01 \\
\hline 1 & 334.9 & 240.2 & 240.1 & -0.04 & 88.8 & 80.4 & 80.3 & -0.20 \\
\hline 2 & 358.7 & 401.1 & 401.5 & 0.10 & 93.9 & 96.6 & 96.6 & 0.01 \\
\hline 3 & 379.6 & 231.9 & 231.9 & 0.02 & 99.5 & 85.2 & 85.2 & -0.09 \\
\hline 4 & 396.4 & 194.4 & 194.4 & -0.01 & 105.4 & 82.6 & 82.4 & -0.23 \\
\hline 5 & 409.6 & 814.5 & 816.4 & 0.23 & 111.9 & 129.5 & 129.5 & -0.03 \\
\hline 6 & 421.1 & 251.3 & 251.2 & -0.03 & 119.1 & 100.0 & 100.0 & -0.04 \\
\hline 7 & 433.0 & 929.4 & 928.1 & -0.14 & 127.1 & 150.8 & 150.7 & -0.07 \\
\hline 8 & 447.8 & 258.4 & 258.3 & -0.06 & 136.3 & 111.5 & 111.4 & -0.04 \\
\hline 9 & 466.5 & 368.8 & 368.9 & 0.03 & 146.8 & 135.5 & 135.5 & 0.02 \\
\hline 10 & 490.8 & 815.2 & 816.6 & 0.17 & 158.8 & 182.3 & 182.4 & 0.05 \\
\hline 11 & 520.4 & 349.9 & 349.6 & -0.09 & 172.4 & 148.4 & 148.4 & -0.04 \\
\hline 12 & 554.9 & 254.7 & 254.6 & -0.03 & 187.2 & 133.9 & 133.9 & 0.00 \\
\hline 13 & 593.2 & 472.0 & 473.1 & 0.23 & 203.0 & 186.6 & 186.8 & 0.11 \\
\hline 14 & 633.6 & 6648.9 & 6866.8 & 3.17 & 219.1 & 318.9 & 319.5 & 0.20 \\
\hline 15 & 673.8 & -2531.8 & -2512.5 & -0.77 & 234.9 & 420.4 & 421.2 & 0.19 \\
\hline 16 & 711.4 & 20012.3 & 20156.7 & 0.72 & 249.5 & 376.9 & 377.2 & 0.06 \\
\hline 17 & 745.0 & 1343.2 & 1339.1 & -0.31 & 262.4 & 311.2 & 311.1 & -0.03 \\
\hline 18 & 774.3 & 760.2 & 758.1 & -0.28 & 273.5 & 271.7 & 271.5 & -0.06 \\
\hline 19 & 798.2 & 593.3 & 591.9 & -0.23 & 282.4 & 251.6 & 251.5 & -0.06 \\
\hline 20 & 818.4 & 540.6 & 539.5 & -0.19 & 289.3 & 244.8 & 244.7 & -0.05 \\
\hline 21 & 835.9 & 550.8 & 550.0 & -0.16 & 294.3 & 248.9 & 248.9 & -0.04 \\
\hline
\end{tabular}

${ }^{a}$ From CSE calculations with single, optically active ${ }^{3} \Pi_{u}$ dissociative channel.

${ }^{\mathrm{b}}$ Effective profile indices for the single, optically inactive ${ }^{5} \Pi_{u},{ }^{1} \Pi_{u}$, and ${ }^{3} \Sigma_{u}^{+}$channels are as in Table V. ${ }^{\mathrm{c}}$ From CSE calculations with four simultaneous dissociative channels, only the ${ }^{3} \Pi_{u}$ channel being optically active.

${ }^{\mathrm{d}} 100\left[q_{v}^{\mathrm{eff}}-1 / \Sigma_{\alpha}\left(1 / q_{v \alpha}^{\mathrm{eff}}\right)\right] / q_{v}^{\mathrm{eff}}$.

zian wing in this region, opposite in sense to the near-wing asymmetry.

\section{Sum rules}

In order to verify numerically the sum rules given in Sec. II D, the multichannel CSE calculations used in Sec. III B 1 were compared with appropriate single-channel calculations. Line-center energies [35], FWHM line widths, and $q^{\text {eff }}$ values were then determined from the calculated single-channel and multichannel SR-resonance cross sections by leastsquares fitting constant-parameter Fano profiles.

Single-channel and multichannel predissociation line widths and energy shifts, resulting from zero-background Fano-profile fits for the case of optically inactive continua, are summarized in Tables III and IV, respectively. The energy shifts were determined by comparison with unshifted resonance energies obtained by solving a single-channel CSE for a variety of couplings, and then extrapolating to zero coupling. The width and shift sum rules, $\Gamma_{v}=\Sigma_{\alpha} \Gamma_{v \alpha}$ and $\Delta_{v}=\Sigma_{\alpha} \Delta_{v \alpha}$, are validated numerically in columns 6-8 of Tables III and IV, within typical relative and absolute accuracies of $0.1 \%$ and $0.0005 \mathrm{~cm}^{-1}$, respectively. These find- ings agree with those of Sink and Bandrauk [13] who found, at a somewhat lower level of precision, that the SR predissociation could be treated as the sum of four isolated curve crossings, thus belonging to the weak-coupling limit.

Corresponding fitted single-channel and multichannel effective Fano profile indices are summarized in Table V, demonstrating several examples of predissociation " $q$ reversal" [36] arising ultimately from the rapidly oscillating "width matrix elements" in Eq. (24) which describes the effects of interactions with other resonances. In the case of the $(0,0)$ and $(1,0)$ resonances for the single ${ }^{3} \Sigma_{u}^{+}$dissociative channel, the calculated lines were so narrow that the Fanoprofile fit failed to give an accurate $q_{v \alpha}^{\text {eff }}$. These resonances may be taken to be effectively Lorentzian and not to contribute to the asymmetry sum. The asymmetry sum rule $1 / q_{v}^{\text {eff }}$ $\approx \Sigma_{\alpha}\left(1 / q_{v \alpha}^{\text {eff }}\right)$ is validated numerically in columns 6-8 of Table $\mathrm{V}$, within typical relative and absolute accuracies of $0.3 \%$ and $3 \times 10^{-6}$, respectively, confirming the predictions of Sec. II D.

Illustrative CSE calculations of $q^{\text {eff }}$ values for an optically active ${ }^{3} \Pi_{u}$ continuum are presented in Table VI for two values of the ${ }^{3} \Pi_{u} \leftarrow X$ electronic transition moment, chosen 
so that the contribution of the active continuum to the lineshape asymmetry is comparable with the effective contribution due to interactions with other resonances, and dominant, respectively, while still ensuring that the overall asymmetry is small. The single ${ }^{3} \Pi_{u}$-channel and multichannel $q^{\text {eff }}$ values in Table VI were determined by fitting constantbackground Fano profiles to the corresponding CSE line shapes. Two interesting observations follow from the results in Table VI. First, the single- ${ }^{3} \Pi_{u}$-channel and multichannel $q$ reversals present in the case of optically inactive continua (Table V) disappear rapidly as the optical activity is increased. Thus, the characters of the asymmetry variations with $v$ due to the inherent $q$ value, arising from the active continuum, and due to the effective contribution, arising principally from interactions with other resonances, are qualitatively different. Second, the asymmetry sum rule is also verified in this case of an optically active continuum, within accuracies comparable with the optically inactive case in Table V.

Hence, while it is impracticable to attempt a comprehensive numerical verification, it appears that a third sum rule, relating to the asymmetry, or inverse effective Fano-profile index, must be added to the well-known width and shift sum rules in cases of weak predissociation by either optically inactive or optically active continua.

\section{SUMMARY AND CONCLUSIONS}

In the case of weak diatomic molecular predissociation by noninteracting, optically inactive continuum states, it has been confirmed that the predissociation line shape is better represented by an asymmetric Beutler-Fano profile than by the usual Breit-Wigner or Lorentzian profile. The principal cause of the line-shape asymmetry is quantum interference between the vibrational resonance of interest and other resonances, with energy dependence of the width parameter for the dominant resonance providing a secondary contribution. In addition, a sum rule for the corresponding line-shape asymmetry $\left(\approx 1 / q^{\text {eff }}\right.$, where $q^{\text {eff }}$ is the effective Fano-profile index) has been derived for this type of predissociation in the case of multiple continua. Using single-channel and multichannel coupled Schrödinger-equation calculations for the Schumann-Runge band system of $\mathrm{O}_{2}$, these findings have been verified numerically. Finally, similar line shapes and sum rules have been found to apply in the case of weak predissociation by optically active continua.

The results suggest that modeling of the cross sections of band systems predissociated by optically inactive continua may benefit from the use of the Fano rather than the Lorentzian line shape, especially in cases where the experimental data to be fitted are of high precision and cover a wide dynamic range, i.e., the application is sensitive to the more distant line wings. Of course, if it is necessary to consider the full range of energy between vibrational resonances, then there is no substitute for full CSE modeling of the cross section [12].

\section{ACKNOWLEDGMENTS}

The authors would like to thank Professor H. LefebvreBrion and Professor R. Lefebvre for critical readings of the manuscript, and Dr. F. H. Mies for valuable insight and advice provided during his stay at ANU, and for a critical reading of the manuscript. This work was supported by DEETYA and the ARC.
[1] U. Fano, Phys. Rev. 124, 1866 (1961).

[2] U. Fano and J.W. Cooper, Phys. Rev. 137, A1364 (1965).

[3] F.H. Mies, Phys. Rev. 175, 164 (1968).

[4] For simplicity, the Beutler-Fano profile is referred to as the Fano profile in most of this work.

[5] A.D. Bandrauk and J.P. Laplante, J. Chem. Phys. 65, 2592 (1976).

[6] A.D. Bandrauk and J.P. Laplante, J. Chem. Phys. 65, 2602 (1976).

[7] J.-P. Connerade and A.M. Lane, Rep. Prog. Phys. 51, 1439 (1988).

[8] F.H. Mies and P.S. Julienne (unpublished).

[9] B.R. Lewis, P.M. Dooley, J.P. England, S.T. Gibson, K.G.H. Baldwin, and L.W. Torop, Phys. Rev. A 55, 4164 (1997).

[10] K. Minschwaner, G.P. Anderson, L.A. Hall, and K. Yoshino, J. Geophys. Res., [Space Phys.] 97, 10103 (1992), and references therein.

[11] Voigt profiles have been employed when the effects of Doppler broadening have been included.

[12] B.R. Lewis, S.T. Gibson, L.W. Torop, and D.G. McCoy, Geophys. Res. Lett. 25, 2457 (1998).

[13] M.L. Sink and A.D. Bandrauk, J. Chem. Phys. 66, 5313 (1977).
[14] M.L. Sink and A.D. Bandrauk, Chem. Phys. Lett. 65, 246 (1979).

[15] M.L. Sink, A.D. Bandrauk, and R. Lefebvre, J. Chem. Phys. 73, $4451(1980)$.

[16] The ratio $\sigma_{d}(E) / \sigma_{c}(E)$ is equivalent to $\rho^{2}$, where $\rho$ is the overlap parameter defined by Fano and Cooper [2].

[17] Under the conditions of interest, $q^{2} \gg 1$ and the second term in Eq. (16) is much smaller than the first in the region of the line core. While the energy dependence of the second term may affect the far-wing line-shape asymmetry, under the applicable condition $(e / q)^{2} \ll 1$, this effect is expected to be unimportant. Hence, it is reasonable to neglect the effects of the small energy dependence in the already small background cross-section term.

[18] Henceforth, we consider the terms "line shape" and "asymmetry" to refer to the first term in Eq. (16), ignoring the constant background term.

[19] Equation (19) follows, for example, from Eq. (65) of Ref. [1], or Eq. (50) of Ref. [3], in the case of an optically inactive continuum.

[20] The shifted energy $E_{v}=E_{v 0}+\Delta_{v}(E)$ [see Eq. (2)].

[21] P.S. Julienne and M. Krauss, J. Mol. Spectrosc. 56, 270 (1975). 
[22] P.S. Julienne, J. Mol. Spectrosc. 63, 60 (1976).

[23] B.R. Lewis, L. Berzins, and J.H. Carver, J. Quant. Spectrosc. Radiat. Transfer 36, 209 (1986).

[24] B.R. Lewis, L. Berzins, J.H. Carver, and S.T. Gibson, J. Quant. Spectrosc. Radiat. Transfer 36, 187 (1986).

[25] H. Lefebvre-Brion and R.W. Field, Perturbations in the Spectra of Diatomic Molecules (Academic, Orlando, FL, 1986), p. 56.

[26] Note that this is a model calculation only, performed in order to demonstrate the validity of the described sum rules. A quantitatively accurate description of the SR absorption lines is given in Ref. [27].

[27] B.R. Lewis, S.T. Gibson, and P.M. Dooley, J. Chem. Phys. 100, 7012 (1994).

[28] P.S. Julienne, D. Neumann, and M. Krauss, J. Chem. Phys. 64, 2990 (1976).

[29] J.P. England, B.R. Lewis, S.T. Gibson, and M.L. Ginter, J.
Chem. Phys. 104, 2765 (1996).

[30] B.R. Johnson, J. Chem. Phys. 69, 4678 (1978).

[31] B.R. Lewis, S.S. Banerjee, and S.T. Gibson, J. Chem. Phys. 102, 6631 (1995).

[32] Equal weights were assumed for each data point in the fit.

[33] In the vicinity of the near-Lorentzian lines considered here, the pseudo-Fano and Fano profiles are equivalent. For simplicity, we fit only Fano profiles to the computed resonances.

[34] K. Yoshino, D.E. Freeman, J.R. Esmond, and W.H. Parkinson, Planet. Space Sci. 35, 1067 (1987).

[35] Fitted line-center energies, corresponding to $\epsilon=0$, were used in determining the predissociation line shifts. The line centers, of course, differ from the line maxima for the asymmetric Fano profile.

[36] B. Kim and K. Yoshihara, J. Chem. Phys. 99, 1433 (1993); B. Kim, K. Yoshihara, and S. Lee, Phys. Rev. Lett. 73, 424 (1994). 